\title{
Lipoid Proteinosis
}

National Institute of Neurological Disorders and Stroke (NINDS)

\section{Source}

National Institute of Neurological Disorders and Stroke (NINDS). Lipoid Proteinosis

Information Page.

Lipoid proteinosis (LP) is a rare disease that affects the skin and the brain. Three distinctive features characterize the disease: a hoarse voice, unusual growths on the skin and mucus membranes, and damage to the temporal lobes or hippocampus of the brain. The symptoms of LP may begin as early as infancy with hoarseness or a weak cry, due to growths on the vocal cords. Skin lesions appear sometime in the next 3 years, leaving acne- or pox-like scars on the face, hands, and mucous membranes. The most characteristic symptom of LP is waxy, yellow, bead-like bumps along the upper and lower edges of the eyelids. Brain damage develops over time and is associated with the development of cognitive abilities and epileptic seizures. Damage to the amygdala, a part of the brain that regulates emotions and perceptions, leads to difficulties in discriminating facial expressions and in making realistic judgments about the trustworthiness of other people. LP is a hereditary disease that equally affects males and females. Nearly a quarter of all reported cases have been in the Afrikaner population of South Africa, but the disease is increasingly being reported from other parts of the world including India. The gene responsible for LP has recently been identified. It performs an unknown function in the skin related to the production of collagen. 